\section{PLAN DE MARKETING INTEGRAL PARA LA COOPERATIVA DE AHORRO Y CRÉDITO SAN JOSÉ LTDA.}

\author{
COMPREHENSIVE MARKETING PLAN FOR COOPERATIVA DE \\ AHORRO Y CRÉDITO SAN JOSÉ LTDA.
}

\author{
PLANO DE MARKETING INTEGRAL PARA COOPERATIVA DE \\ AHORRO E CRÉDITO SAN JOSÉ LTDA.
}

\section{Grace Alexandra Velasteguí Bósquez y Elsa Elisa Estrada Miño}

Artículo recibido julio 2021 | Arbitrado agosto 2021 | Aceptado septiembre 2021 | Publicado 13 de diciembre 2021

\section{Resumen}

Se realizó un estudio en la entidad financiera Cooperativa San José Ltda., con el objetivo de proponer un Plan de Marketing Integral que permita acrecentar la rentabilidad social y financiera del lugar en estudio. Basada en una metodología aplicada, con un diseño, documental de campo; no experimental. Los enfoques aplicados fueron cualitativo y cuantitativo, los métodos empleados fueron inductivo, deductivo, analítico, sintético y sistemático. Se utilizó como técnica la entrevista estructurada y la etnografía. La muestra, se conformó por el gerente general, jefe de negocios y jefe de marketing, en caso de los socio, 16 representantes de la institución. El resultado se centró en la necesidad de actualizar y mejorar el plan de marketing en referencia a la dinámica actual del mercado. Se concluye que con la propuesta se fortalece la cultura institucional, medios de comunicación tradicionales y digitales, así que, se recomienda la aplicabilidad del Plan de Marketing Integral.

Palabras clave: Marketing; marketing integral; marketing servicios, plan marketing

\begin{abstract}
A study was carried out in the financial entity Cooperativa San José Ltda., With the aim of proposing a Comprehensive Marketing Plan that allows increasing the social and financial profitability of the place under study. Based on an applied methodology, with a design, field documentary; not experimental. The applied approaches were qualitative and quantitative, the methods used were inductive, deductive, analytical, synthetic and systematic. The structured interview and ethnography were used as a technique. The sample was made up of the general manager, business manager and marketing manager, in the case of partners, 16 representatives of the institution. The result focused on the need to update and improve the marketing plan in reference to the current market dynamics. It is concluded that the proposal strengthens the institutional culture, traditional and digital media, so the applicability of the Comprehensive Marketing Plan is recommended.
\end{abstract}

Key words: Marketing; comprehensive marketing; marketing services, marketing plan
Grace Alexandra Velasteguí Bósquez gracealexandravelastegui@gmail.com Orcid: 0000-0001-6391-9733

Escuela Superior Politécnica de Chimborazo, Riobamba-Ecuador.

Magister en Marketing y Servicio al Cliente de la Escuela Superior Politécnica de Chimborazo, Ingeniera en Comercio Exterior, Experiencia Laboral: Docente Investigadora y miembro del tribunal de tesis de pregrado de la Universidad Estatal de Bolivar.

Elsa Elisa Estrada Miño

elyestrada7@gmail.com

Orcid: 0000-0002-3503-4003

Universidad Estatal de Bolívar - Ecuador.

Ingeniera en Comercio Exterior Mención en Negociaciones Internacionales, Escuela Superior Politécnica de Chimborazo. Magister en Marketing y Servicio al Cliente. Experiencia en Administración estatal Pública en el área financiera del BanEcuador, Docente Investigadora y miembro del tribunal de varias tesis de pregrado, de la Universidad Estatal de Bolívar y Conferencista en empresas públicas y privadas del Ecuador y Perú. Universidad Estatal de Bolivar, Ecuador. 


\section{Resumo}

Foi realizado um estudo na entidade financeira Cooperativa San José Ltda., com o objetivo de propor um Plano Integral de Marketing que permita aumentar a rentabilidade social e financeira do local em estudo. Com base numa metodologia aplicada, com um documentário de design e de campo; não experimental. As abordagens aplicadas foram qualitativas e quantitativas, os métodos utilizados foram indutivos, dedutivos, analíticos, sintéticos e sistemáticos. A entrevista estruturada e a etnografia foram utilizadas como técnica. A amostra foi composta pelo gerente geral, gerente comercial e gerente de marketing, no caso de sócios, 16 representantes da instituição. O resultado focou na necessidade de atualização e aprimoramento do plano de marketing tendo em vista a atual dinâmica do mercado. Conclui-se que a proposta fortalece a cultura institucional, tradicional e mídia digital, portanto, recomenda-se a aplicabilidade do Plano Integral de Marketing.

Palavras chave: Marketing; marketing abrangente; serviços de marketing, plano de marketing

\section{INTRODUCCIÓN}

Es marketing se conceptualiza el como un proceso mediante el cual se diseña, ejecuta la fijación de promociones, precios y todo aquello que se encuentra relacionado con los productos o servicios que oferta una empresa, con el fin de que se cree intercambios que satisfagan las necesidades de las personas según manifiesta López (2001). También, es considerado como un proyecto social y administrativo mediante el cual los individuos y las organizaciones obtienen lo que necesitan y desean creando e intercambiando valor con otros, (Armstrong, 2003).

Es así ue se puede conceptualizar al marketing como un proceso de cohesión entre consumidores y empresas con el objetivo de proporcionar los medios adecuados para que éstos se produzcan, éste término hace alusión a la actividad que va más allá de únicamente vender productos y servicios, es un proceso bidireccional en donde la empresa ofrece lo que el cliente desea, así también es una actividad a largo plazo que exige disponer de información sobre las necesidades y deseos de los clientes, pero sin olvidarse del entorno competitivo en el que opera la empresa.

Por ello, el marketing en la actualidad, es un accionar importante de comunicación comercial a la que varias empresas destinan mayores recursos económicos, incluso sobrepasando la inversión publicitaria en medios convencionales, es así que el nuevo modelo de marketing se focaliza enel manejo adecuado y cuidadoso de la relación existente entre el cliente con la institución, por ello, disponer de información sobre los clientes, características, necesidades y preferencias es fundamental para el éxito de cualquier organización.

Según Donald y Gray (2004) especifican que la importancia del marketing radica en el proceso de examinar la ventaja competitiva en el mercado para la aplicación de estrategias que permitan cumplir la satisfacción del cliente de manera específica a través de los productos y servicios que una empresa oferta.

A demás, Kotler, (2006) menciona que "la dirección del marketing está basado en la satisfacción de los deseos de las personas, siendo una de las funciones más representativas de la empresa el ofrecer un producto o servicio a cambio de la obtención de una rentabilidad beneficiosa para la organización". (s/p). Todas las actividades dentro de una empresa requieren del esfuerzo del ser humano, de tal manera que se puedan crear productos $y$ 
otorgar servicios pensando en las necesidades, sin embargo, el cliente es el promotor que la empresa funcione ya que las actividades van en dirección a ellos.

De esta manera, el marketing de servicios para Balseca (2013), se considera como un conjunto de estrategias de mercado en donde se ofertan y comercializan productos intangibles, los mismos que están dirigidos hacia la satisfacción de la demanda con el beneficio de la obtención económica"

Por otro lado, la evolución de la tecnología permite que los sistemas de información estén cerca de las instituciones y el ser humano, sin embargo en muchas organizaciones los procesos y estilos de comunicación externa se han ido quedando, utilizando muchas de las veces procesos tradicionales o poco adecuados, que no representan una forma clara de venta que maximice beneficios para la institución, por tanto es importante realizar el estudio de investigación sobre los procesos de comunicación interna y externa, como el nivel de satisfacción que deben tener los socios y clientes de la institución.

La entidad financiera Cooperativa de Ahorro y Crédito San José Ltda. es controlada por la Superintendencia de Economía Popular y Solidaria, el cual pertenece al segmento 1, y oferta productos, servicios financieros y no financieros a socios y clientes, lo que le ha logrado posicionamiento en su área de influencia.

Cabe resaltar, que en la actualidad las estrategias de venta que maneja la Institución a través de su Plan de Marketing son pocas adecuadas, debido a que no llegan a la totalidad de sus socios y clientespor un lado y por otro, la forma expresiva en el lenguaje es poco receptiva ya que mantiene frases de difícil entendimiento para la mayoría de sus asociados por su condición de ruralidad y educación; así como características de difícil comprensión; es así que la no identificación de estas particularidades no le permiten mejorar los canales de comunicación y venta para ofrecer una experiencia diferente de marca y maximizar su renta social y financiera.

Por tal motivo, es importante reconocer el quehacer institucional frente a lossegmentos poblacionales que requieren sus productos y servicios, para proponer herramientas de gestión altamente efectivas que permitan acrecentar su posicionamiento y sostenibilidad con responsabilidad social. Por ello, surge una interrogante ¿Con la implementación del Plan de Marketing Integral se acrecentará la rentabilidad social y financiera para la institución? La cual se buscar responder con el objetivo general, el cual, consiste en la propuesta de un Plan de Marketing Integral que permita acrecentar la rentabilidad social y financiera de la Cooperativa de Ahorro y Crédito San José Ltda.

El Plan de Marketing Integral para la Cooperativa de Ahorro y Crédito San José Ltda., del Cantón Chimbo, Provincia Bolívar, representa una importante herramienta de gestión, ya que su definición permitirá alcanzar un posicionamiento de marca, generar expectativa de demanda de productos y servicios que la institución oferta y con ello maximizar su rentabilidad social y financiera. Por ello, es importante que la institución 
centralicelafiguradelsociocomo parteesencial de éxito, debiendo enfocar sus objetivos en la búsqueda y satisfacción de las necesidades y expectativas de los mismos, analizando a cada uno de los segmentos de mercado, en relación a su área de influencia, pues sus necesidades variaran conforme sus características. Además, adoptar las estrategias como herramienta de gestión, traducido en un Plan de Marketing Integral, ayudará que la institución llegue de forma personalizada con potenciales clientes, identificar sus necesidades, segmentar mercados e inclusive reformular sus productos y servicios financieros acorde a las exigencias del mercado.

De la misma manera, el desarrollo del tema investigativo permite tener mayor conocimiento del marketing integral, debido a que está aplicado a las reales necesidades, lo que ayudará a generar ingresos y mejor posicionamiento en el mercado. Además, es pertinente porque se cuenta con los conocimientos teóricos y metodológicos para el proceso de la investigación, así como de los recursos necesarios.

Se toma en cuenta para el estudio los términos relacionados con el Plan de Marketing. De esta forma, se inicia con la conceptualización del mismo, por Robbins (2013), el cual indica que el plan de marketing no es más que un documento que es estructurado con el fin de poner en práctica una planificación estratégica de la organización. Se puede decir entonces, que el plan de marketing es un documento escrito en el que, de forma sistemática y estructurada, y previa realización de los correspondientes análisis y estudios, se definen los objetivos a conseguir en un período de tiempo determinado, así como se detallan programas y medios de acción precisos para alcanzar los objetivos enunciados en el plazo previsto.

De acuerdo lo establecido por Gary y Kotler, el Plan de Marketing es la herramienta básica de gestión que toda empresa debe utilizar, la misma está orientada al mercado que quiera ser competitivo. En su puesta en marcha quedarán fijadas las diferentes actuaciones que deben realizarse en el área del marketing, para alcanzar los objetivos marcados. (Gary y Kotler, 2013).

Por ende, en una actividad como la del marketing, todas las acciones que se realicen deben ser ejecutadas con la debida planificación, ya que al hacerlo se tiene una gran ventaja de no llegar al fracaso o al menos tener una manera de como ejecutarlo y no concurrir al gasto de recursos y esfuerzos. A su vez, Bernal (2014), señala que es importante para que una empresa tenga éxito de manera que se cumpla con todos los objetivos y visiones de la empresa, por lo que las acciones del marketing se identifican claramente con la realización de la publicidad o de la comunicación.

Mientras tanto, el Marketing Integral, es un enfoque de marketing que define la necesidad de unificar e integrar todos los canales de comunicación para ofrecer una experiencia de marca coherente para el consumidor. Es así como Herrera (2006), menciona que la mercadotecnia integral o también llamado marketing integral es una disciplina que integra una serie de factores que debe tomar 
en cuenta o ser consideradas por las empresas para obtener el éxito necesario para continuar operando conforme a las metas y objetivos desde su planeación estratégica con las mejores medidas, prácticas y herramientas para llevarla a buen término. Su importancia radica en el manejo adecuado de las herramientas ante la generación de publicidad las ventas de un producto o servicio, ya que, depende en gran parte de la situación de cada producto y/o mercado. (Costa, 2014).

\section{MÉTODO}

La metodología de la investigación que se utilizó fue de tipo aplicada, ya que, ayudó a dar una solución eficiente al problema que se identificó en el centro de estudio basada en los resultados del estudio. Con un diseño, documental de campo; porque se analizaron boletines de información institucional, y directamente en el lugar objeto de estudio. De esta forma, fue una investigación no experimental.

Su enfoque, estuvo caracterizado por ser cualitativo porque es de carácter social; permitió establecer la relación entre el sujeto de estudio y el entorno mediante entrevistas a funcionarios como también la etnografía a socios y clientes; y cuantitativo porque se basó en métodos para la recolección de datos; a través del análisis e interpretación de opiniones comunes agrupadas en tablas con frecuencia absoluta- relativa de la entrevista y etnografía.
Aunado a ello, los niveles de la investigación estuvieron conformado por el exploratorio y el descriptivo. El primero, debido al que el estudio se efectúo en la fase de recopilación de información para obtener el diagnóstico de la situación actual en relación a la gestión de marketing de la institución y sus repercusiones en la renta social y financiera. El segundo, porque con este método se identificó las necesidades en base a los resultados de la investigación, para elaborar el Plan de Marketing Integral de la Cooperativa de Ahorro y Crédito San José Ltda., y la receptividad que se espera del mismo. A su vez, los métodos desarrollados en el estudio fueron inductivo, deductivo, analítico, sintético y sistemático.

De esta forma, se utilizaron como técnicas para la recolección de datos, la observación no participativa; esta técnica permitió conocer de cerca la realidad de la institución sobre los procesos de marketing institucional y la difusión de sus servicios, permitiendo identificar fortalezas y debilidades de manera oportuna. La entrevista estructurada, la cual le fue aplicada a la Gerencia General, Jefe de Negocios y Jefe de Marketing y sirvió para identificar cómo está la institución en el ámbito de marketing, sicuenta con estrategias efectivas y parámetros relevantes para el desarrollo de la investigación. Y la etnografía; ésta técnica permitió que los socios y clientes, expusieran su percepción y necesidades a acerca de la receptividad del mensaje institucional, que mediante las técnicas de marketing se han 
empleado para la socialización o difusión de los servicios y productos financieros y no financieros de la entidad. Mientras que los instrumentos utilizados fueron, guía y registro de observación, guía de entrevista, y ficha o guía etnográfica.

Cabe destacar, que el centro de estudio estuvo representado por la Cooperativa de Ahorro y Crédito San José Ltda., del Cantón Chimbo, Provincia Bolívar; lugar donde se seleccionó la población, la cual fue representada por socios, clientes y demás funcionarios de la identidad financiera. $Y$ la muestra, en caso de los funcionarios se realizó la entrevista al gerente general, jefe de negocios y jefe de marketing, en caso de los socio, se entrevistaron a 16 representantes de la institución.

\section{RESULTADOS}

\section{Entrevista}

La aplicación de esta técnica partió de la estructuración de la ficha de la entrevista, misma que se fundamentó en las perspectivas que en la ficha metodológica se estableció a fin de recabar información importante para establecer el diagnóstico del problema planteado en este estudio. De esta manera, la entrevista fue aplicada al Gerente General, Jefe de Negocios y Jefe de Marketing. La misma estuvo conformada por siete preguntas, cuyas respuestas fueron si, a veces o no. (Tabla 1 ).

Tabla 1. Cuestionario de la entrevista aplicado a los funcionarios de la institución.

\begin{tabular}{|c|c|c|c|c|}
\hline INTERROGANTES & SI & A VECES & NO & TOTAL \\
\hline ¿Conoce e identifica claramente el segmento de mercado? & 3 & & & 3 \\
\hline $\begin{array}{l}\text { ¿La institución difunde asertivamente los productos y } \\
\text { servicios financieros y no financieros? }\end{array}$ & 3 & & & 3 \\
\hline $\begin{array}{l}\text { ¿Sus colaboradores conocen los productos y servicios que } \\
\text { oferta la institución? }\end{array}$ & 2 & 1 & & 3 \\
\hline $\begin{array}{l}\text { ¿La receptividad del mensaje de los medios publicitarios } \\
\text { llega de forma adecuada a sus socios y clientes? }\end{array}$ & 2 & 1 & & 3 \\
\hline $\begin{array}{l}\text { ¿Ha determinado el costo beneficio de la inversión en } \\
\text { publicidad? }\end{array}$ & & & 3 & 3 \\
\hline $\begin{array}{l}\text { ¿Las estrategias de marketing actual ayudan a acrecentar la } \\
\text { renta social y financiera de la institución? }\end{array}$ & & & 3 & 3 \\
\hline $\begin{array}{l}\text { ¿La Cooperativa cuenta con un Plan de Marketing Integral } \\
\text { adaptado a sus necesidades? }\end{array}$ & & 3 & & 3 \\
\hline TOTAL & 10 & 5 & 6 & 21 \\
\hline
\end{tabular}


En la Tabla 2 y Figura 1 se destacan los resultados obtenidos de la entrevista realizada a los 3 funcionarios de la Cooperativa de Ahorro y Crédito San José Ltda. (Gerente General, Jefe de Negocios y Jefe de Marketing.), a través de un análisis descriptivo en el cual se pudo evidenciar que la mayoría de respuestas correspondieron a la opción "Si" con un $47,62 \%$ del total entrevistado y un $23,81 \%$ que responden según el criterio de evaluación "A veces", y el porcentaje de incumplimiento es significante o numéricamente elevado por tener un $28,57 \%$ correspondiente a la opción "No".

Tabla 2. Resultados acumulados de la entrevista aplicada a funcionarios de la institución.

\begin{tabular}{|c|c|c|}
\hline ALTERNATIVAS & FRECUENCIAS & PORCENTAJES \\
\hline SI & 10 & $47,62 \%$ \\
\hline A VECES & 5 & $23,81 \%$ \\
\hline NO & 6 & $28,57 \%$ \\
\hline TOTAL & 21 & $100 \%$ \\
\hline
\end{tabular}

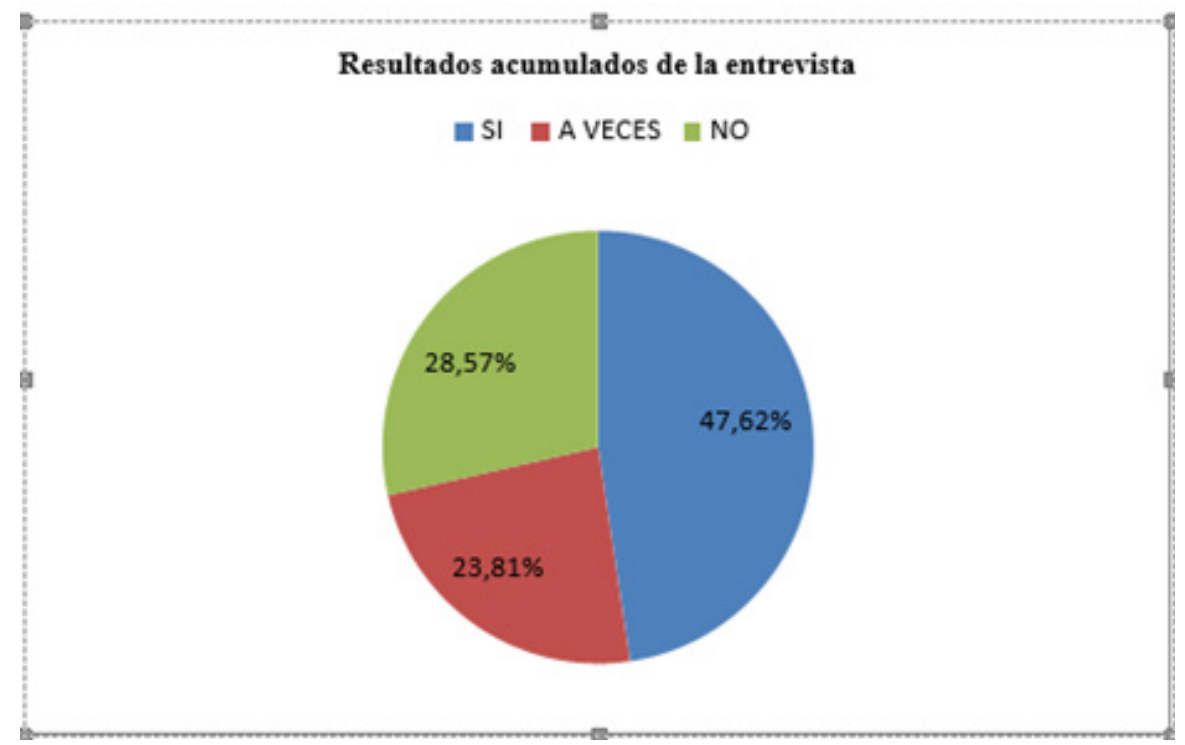

Figura 1. Resultados acumulados de la entrevista aplicada a funcionarios de la institución.

\section{Etnografía}

La etnografía fue aplicada y desarrollada a lossociosyclientesque demandandeproductos y servicios financieros y no financieros de la Cooperativa San José Ltda., realizado en las oficinas de Chillanes, Montalvo y Ventanas, oficinas estratégicamente seleccionadas, se obtuvieron los siguientes resultados. La misma estuvo conformada por siete preguntas, cuyas respuestas fueron si, a veces o no. (Tabla 3). 
Tabla 3. Cuestionario de la etnografía aplicada a socios y clientes de la institución.

\begin{tabular}{|c|c|c|c|c|}
\hline INTERROGANTES & SI & MUY POCO & NO & TOTAL \\
\hline ¿Asiste regularmente a la institución? & 10 & 2 & 4 & 16 \\
\hline $\begin{array}{l}\text { ¿Conoce los productos y servicios financieros y no financieros que } \\
\text { ofrece la institución? }\end{array}$ & 3 & 12 & 1 & 16 \\
\hline $\begin{array}{l}\text { ¿Ha recibido información clara de parte de los funcionarios de la } \\
\text { institución en relación a su oferta? }\end{array}$ & 2 & 5 & 9 & 16 \\
\hline $\begin{array}{l}\text { ¿Conoce los medios publicitarios que utiliza la institución para } \\
\text { difundir los servicios y productos financieros? }\end{array}$ & 1 & 5 & 10 & 16 \\
\hline $\begin{array}{l}\text { ¿Considera usted que es de fácil entendimiento el lenguaje } \\
\text { para publicitar la oferta institucional? }\end{array}$ & 2 & 7 & 7 & 16 \\
\hline $\begin{array}{l}\text { ¿Piensa usted que los medios publicitarios que utiliza la institución } \\
\text { son de fácil accesibilidad? }\end{array}$ & 1 & 5 & 10 & 16 \\
\hline $\begin{array}{l}\text { ¿Conoce usted si la institución utiliza medios digitales para ofertar } \\
\text { sus servicios financieros? }\end{array}$ & 1 & 6 & 9 & 16 \\
\hline TOTAL & 20 & 42 & 50 & 112 \\
\hline
\end{tabular}

En la Tabla 4 y Figura 2 de la etnografía realizada a los 16 socios y clientes de la Cooperativa de Ahorro y Crédito San José Ltda., son mostrados los resultados del análisis descriptivo en el cual se pudo evidenciar que la mayoría de respuestas correspondieron a la opción "No" con un 44,625\% del total de participantes y un $37,50 \%$ que responden según el criterio de evaluación "Muy poco", y el porcentaje numéricamente bajo es 17,85\%correspondiente a la opción "Si".

Tabla 4. Resultados acumulados de la etnografía aplicada a socios y clientes de la institución.

\begin{tabular}{lcc}
\hline \multicolumn{1}{c}{ ALTERNATIVAS } & FRECUENCIAS & PORCENTAJES \\
\hline SI & 20 & $17,85 \%$ \\
MUY POCO & 42 & $37,50 \%$ \\
NO & 50 & $44,65 \%$ \\
\hline TOTAL & $\mathbf{1 1 2}$ & $\mathbf{1 0 0 \%}$ \\
\hline
\end{tabular}




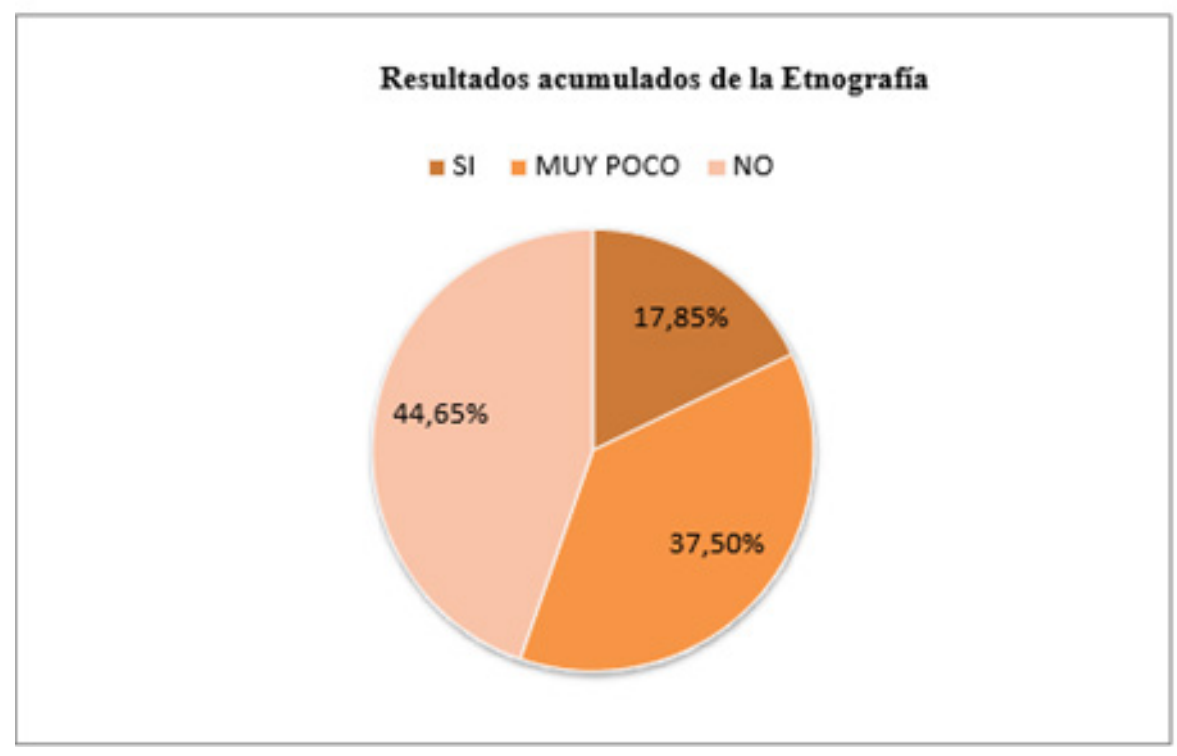

Figura 2. Resultados acumulados de la etnografía aplicada a socios y clientes de la institución.

\section{Análisis}

Identificadaslas perspectivas delaGerencia General, Jefatura de Negocios y Jefatura de Marketing, manifestaron tener identificado el segmento de mercado de la institución. Por otro lado, los grupos entrevistados como gerente, jefe de negocios y de marketing, así como los empleados de la cooperativa, señalaron que todos conocen sobre los beneficios y ofertas que tiene la institución, en contradicción con lo que señalan los clientes y socios que indicaron que no tienen información precisa sobre los beneficios que presta la institución por parte de los empleados de la misma.

A pesar que los directivos manifestaron que se difunde en los diferentes medios de comunicación sobre los servicios financieros de manera clara, se pudo identificar que los socios y clientes, señalan que las estrategias utilizadas; como la receptividad del mensaje que se difunde en los medios de comunicación no es la adecuada, pues no llegan de manera directa al socio y cliente por factores de cobertura geográfica y lenguaje claro para el tipo de receptor que tiene el mensaje. Es así que se reconoce la necesidad de actualizar y mejorar el plan de marketing en referencia a la dinámica actual del mercado. (cultura institucional, medios de comunicación tradicionales y publicidad digital).

Propuesta Plan de Marketing Integral para la Cooperativa de Ahorro y Crédito San José Ltda., del Cantón Chimbo, Provincia Bolívar

Partiendo del diagnóstico realizado en base a la utilización de las técnicas metodológicas que identificaron las falencias que existe en el Plan de Marketing actual de la Cooperativa San José Ltda., se plantean estrategias orientadas a satisfacer las necesidades de los socios y clientes, garantizando la efectividad y transparencia en los procesos como parte de la responsabilidad social. Las Estrategias se orientan en fortalecer tres pilares fundamentales, las mismas que se describen en la Figura 3. 


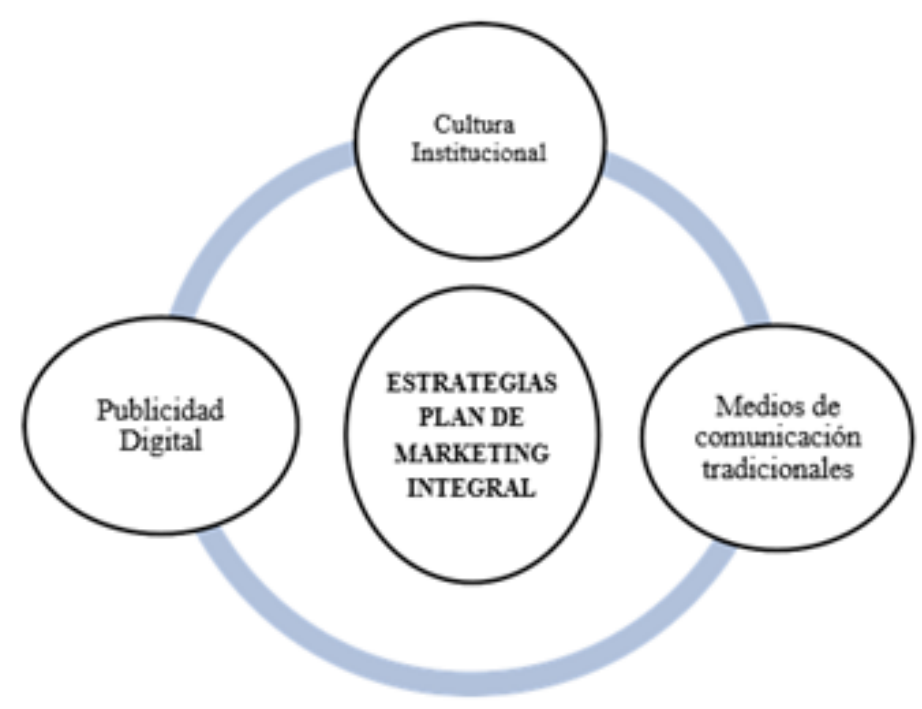

Figura 3. Estrategias del Plan de Marketing Integral

\section{Primera estrategia. Cultura institucional}

Tabla 5. Estrategia cultura institucional.

Objetivo

Acciones a ser ejecutadas
Promover el desarrollo de las personas, mejorando la interacción entre las mismas, así como la calidad de los productos y servicios.

- Atención personalizada.

- Empoderamiento del portafolio de productos y servicios por parte de funcionarios y colaboradores de la institución.

- Mantener un lenguaje claro y de fácil entendimiento para socios y clientes de la entidad.

- Coherencia con los valores corporativos.

- Selección del talento humano.

- Estilos de vida saludables.

\section{Responsables}

\section{Gerencia General}

Consejo de Administración y Vigilancia.

\section{Beneficiarios}

Funcionarios y Colaboradores de la Institución.

Socios y Clientes 


\section{Segunda estrategia. Medios de comunicación Offline (tradicionales)}

Tabla 6. Estrategia Medios de comunicación Offline (tradicionales).

\begin{tabular}{lll}
\hline Objetivo & - & $\begin{array}{l}\text { Informar constantemente a todo el segmento de mercado sobre la oferta de } \\
\text { productos y servicios financieros y no financieros de la institución. }\end{array}$ \\
Acciones a ser ejecutadas & - & Dar a conocer, las actividades, los productos y servicios financieros y no finan- \\
& cieros de la institución.
\end{tabular}

\section{Tercera estrategia. Medios de comunicación online (digitales)}

Tabla 7. Estrategia Medios de comunicación online (digitales).

\begin{tabular}{lll}
\hline Objetivo & - & Aprovechar el impacto que tienen las redes sociales para difundir la imagen y \\
& crear mayor fidelidad de la Cooperativa de Ahorro y Crédito San José Ltda.
\end{tabular}




\section{Plan Marketing Integral Semestral, Cronograma y recursos}

Tabla 8. Plan de Marketing Integral Semestral, Cronograma, Recursos.

\begin{tabular}{|c|c|c|c|c|c|c|c|c|}
\hline \multirow{2}{*}{ ESTRATEGIAS } & \multirow{2}{*}{ ACCIONES } & \multirow{2}{*}{ RECURSOS } & \multicolumn{6}{|c|}{ AÑo $x x x x$} \\
\hline & & & Julio & Agosto & Septiembre & Octubre & Noviembre & Diciembre \\
\hline \multirow[t]{6}{*}{ Cultura Institucional } & Atención personalizada & Humano & & & & & & \\
\hline & $\begin{array}{l}\text { Empoderamiento del portafolio de productos y servicios por } \\
\text { parte de funcionarios y colaboradores }\end{array}$ & $\begin{array}{l}\text { Humano } \\
\text { Tecnológicos }\end{array}$ & & & & & & \\
\hline & $\begin{array}{l}\text { Mantener un lenguaje claro y de fácil entendimiento para } \\
\text { socios y clientes de la entidad. }\end{array}$ & Humano & & & & & & \\
\hline & Coherencia con los valores corporativos. & Humano & & & & & & \\
\hline & Selección del talento humano. & Humano & & & & & & \\
\hline & Estilos de vida saludables. & Humano & & & & & & \\
\hline $\begin{array}{l}\text { Medios de comunicación } \\
\text { offline (tradicionales). }\end{array}$ & $\begin{array}{l}\text { Dar a conocer, las actividades, los productos y servicios } \\
\text { financieros y no financieros de la institución. }\end{array}$ & $\begin{array}{l}\text { Humano } \\
\text { Tecnológicos }\end{array}$ & & & & & & \\
\hline \multirow[t]{4}{*}{$\begin{array}{l}\text { Medios de comunicación } \\
\text { online (digitales). }\end{array}$} & Crear la cuenta oficial en Facebook (fan page). & $\begin{array}{l}\text { Humano } \\
\text { Tecnológicos }\end{array}$ & & & & & & \\
\hline & Crear la cuenta oficial en Twitter. & $\begin{array}{l}\text { Humano } \\
\text { Tecnológicos }\end{array}$ & & & & & & \\
\hline & Actualizar la página web de la institución. & $\begin{array}{l}\text { Humano } \\
\text { Tecnológicos }\end{array}$ & & & & & & \\
\hline & $\begin{array}{l}\text { Realizar un control semanal del seguimiento a la publicidad } \\
\text { digital. }\end{array}$ & $\begin{array}{l}\text { Humano } \\
\text { Tecnológicos }\end{array}$ & & & & & & \\
\hline
\end{tabular}


Validación de las estrategias por el método de expertos

Para validar las estrategias propuestas para el diseño del Plan de Marketing Integral en la Cooperativa de Ahorro y Crédito San José Ltda., se procede a la implementación del método de expertos. Se preseleccionan 10 personas con conocimientos y experiencia sobre el Plan de Marketing Integral de instituciones financieras de la Provincia Bolívar. Sin embargo, solamente se seleccionaron 7 personas.

\section{CONCLUSIONES}

Se realizó el diagnóstico situacional de la Cooperativa San José Ltda., enfocado en los diferentes grupos de interés, como son los funcionarios, socios y clientes, donde se obtuvo información relevante sobre la gestión del Plan de Marketing Integral, para buscar alternativas de solución a la problemática institucional. Por lo tanto, se reconoce la necesidad de actualizar y mejorar el plan de marketing en referencia a la dinámica actual del mercado; esto, en virtud de maximizar los beneficios buscando la satisfacción de las necesidades de sus socios y clientes por un lado y por otro garantizar la sostenibilidad del negocio.

Por ende, se propone estrategias para fortalecer la cultura institucional, medios de comunicación tradicionales y digitales, plasmadas en un Plan de Marketing integral, que ayudaran a acrecentar la renta social y financiera.

Por último, se realizó la validación de expertos que fueron sido seleccionados previo un análisis de su experiencia y profesionalismo, los mismos que validaron el Plan de Marketing Integral propuesto para la institución, como también a ser aplicados en otras instituciones financieras. De este modo, se recomienda la aplicabilidad del Plan de Marketing Integral para la Cooperativa San José Ltda.

\section{REFERENCIAS}

Armstrong, K. (2003). Fundamentos del marketing sexta edición. México

Balseca, W. (2013). Diseño de un plan de marketing . Recuperado de https://dspace. ups.edu.ec/bitstream/123456789/4666/1/ UPS-QT03635.pdf

Bernal, B. (2014). Posicionamiento en el mercado. México

Costa, P. (2014). Agencia marketing integral . Recuperado de: http://www.notonlywebs. com/social-media/turismo-y-redessociales/

Donald, C., y Gray, D. (2004 ). Marketing en la pequeña y mediana empresa. Bogotá: Norma

Gary, A., y Kotter, P. (2013). Fundamentos del marketing. Pearson Educación

Herrera, J. (2006). La mercadotecnia integral en la actualidad. Recuperado de https:// jorgeherreraga.wordpress.com/lamercadotecnia-integral-en-la-actualidad/

Kotler, P. (2006). El marketing. Canadá : Universidad Australiana

López, B. (2001). La esencia del marketing. Barcelona: Universidad Politécnica de Cataluña

Robbins, S. (2013). Comportamiento Organizacional. México: Pearson Educación 